in this gene are also associated with schizophrenia and BD. An hypothetical model of shared mechanisms between bVFTD and BD was proposed, including specific mendelian mutations associated with genetic predisposition (e.g. brain-derived neurotrophic factor-BDNF gene) and environmental factors with an effect on cellular homeostasis (e.g. increased cell deaths, decreased synthesis of synaptic proteins) and an influence over behavioural and cognitive symptoms. Nevertheless, comparison of the executive functions, social cognition profiles and structural neuroimaging of bVFTD and elderly patients with BD showed difference in patterns.

Discussion: Although BD is principally considered a neurodevelopment disorder, while FTD is a neurodegenerative disorder, follow-up studies of cognitive deficits, imaging, and genetics in BD patients could elucidate the possible correlation between these major diseases and may have implications for pathogenesis, as well as for treatment.

\title{
435 - Innovation and entrepreneurship in gerontology and psychogerontology. The INVENTHEI project.
}

Prof. David Facal, Raquel Rodriguez Gonzalez, Cristina Lojo-Seoane, Manuel Gandoy-Crego

INVENTHEI (INnoVation and ENTrepreneurship in Higher Education Institutions) is an European project aimed to enhance the regional innovation ecosystems and promote innovation-driven research. In Spain, the University of Santiago de Compostela participates through the Master's Degree in Psychogerontology (Faculty of Psychology) and the Master's Degree in Gerontology (Faculty of Nursing). Learning and mentoring programme related with the ageing process is presented, focused on the social innovation and transfer capacities of our students, faculty and staff members. A challenged based multidisciplinary learning method is followed, including three training sessions (Session 1: Person- centred innovation; Session 2: Team-centred innovation; Session 3: Results-centred innovation) and online mentoring. Using this methodology, transfer of the learning is promoted in an agile and practical

way through the resolution of challenges proposed by external partners in the sector of gerontology and healthcare. The social innovation-training programme is complemented with a workshop about innovation driven research in aging and presentation of business cases, involving the organizational ecosystem of the sector.

\section{6 - Scaling up a community-based intervention for people affected by dementia: what is the value? \\ Nathan Stephens}

The Meeting Centres Support Programme [MCSP] provides community-based social, emotional and practical support for people affected by dementia to adjust to the changes dementia brings. Since development in the Netherlands the MCSP has been successfully adapted and implemented in the UK led by the Association for Dementia Studies, University of Worcester. In January (2020), Worcestershire County Council announced $£ 540,000$ to scale up the provision of MCSPs across the county: Worcestershire Meeting Centres Community Support Programme [WMCCSP].

The novel county-wide approach will build real capacity, increasing the amount of people accessing post-diagnostic support, integrating services, reducing inequalities, and improving health and wellbeing; fundamental to the COVD-19 recovery plan (Department of Health and Social Care, 2020). This raises questions about the type of 'value' interventions such as the WCCMCSP should seek to achieve, including how it is captured and measured (Redding, 2016). This becomes more relevant when recognising only a 